\title{
High-sensitivity cardiac troponin $T$ is a predictor of recurrent acute coronary syndrome in patients with acute myocardial infarction
}

\author{
S.-C. Sheng' ${ }^{1}$ X.-M. Lu' ${ }^{1}$, Y. Kang ${ }^{1}$, Y.-P. Du ${ }^{2}$ and G.-X. Wang ${ }^{3}$ \\ ${ }^{1}$ Department of Clinical Laboratory, the Second People's Hospital of Yibin City, \\ Sichuan, Province, China \\ ${ }^{2}$ Department of Cardiology, the Second People's Hospital of Yibin City, \\ Sichuan Province, China \\ ${ }^{3}$ Applied Biology Research Center, College of Bioengineering, \\ Chongqing University, Chongqing, China \\ Corresponding author: G.-X. Wang \\ E-mail: guixuewang199@163.com \\ Genet. Mol. Res. 13 (2): 3819-3825 (2014) \\ Received July 12, 2013 \\ Accepted December 16, 2013 \\ Published May 16, 2014 \\ DOI http://dx.doi.org/10.4238/2014.May.16.6
}

\begin{abstract}
High-sensitivity cardiac troponin $\mathrm{T}$ is a useful tool for diagnosing myocardial ischemia. However, its role in the prognosis of patients with acute myocardial infarction has not been studied. Here, the prognostic value of high-sensitivity cardiac troponin $\mathrm{T}$ for patients with acute myocardial infarction was investigated. The concentrations of high-sensitivity cardiac troponin $\mathrm{T}$, other clinical chemistry makers, and living habits were investigated at the time of admission in patients with acute coronary syndrome, whereas the high-sensitivity cardiac troponin $\mathrm{T}$ concentrations at $6 \mathrm{~h}$ after admission and during recovery were analyzed in other patient groups. The concentration of high-sensitivity cardiac troponin $\mathrm{T}$ was significantly higher in patients with acute myocardial infarction than in those with other cardiac diseases and in controls ( $\mathrm{P}$ $<0.01)$. Based on the standard diagnostic criterion, 134 patients were diagnosed with acute myocardial infarction. Monitoring the change in concentration of high-sensitivity cardiac troponin $\mathrm{T}$ in patients with acute
\end{abstract}


coronary syndrome can reduce the risk of recurrence and death.

Key words: Acute coronary syndrome; Acute myocardial infarction; High-sensitivity cardiac troponin T; Prognosis; Living habits

\section{INTRODUCTION}

Acute myocardial infarction (AMI) is the most common and serious cardiovascular disease worldwide. Attempts to find new AMI markers, ranging from the initial myocardial enzymes (aspartate aminotransferase, L-lactate dehydrogenase, creatine kinase, and hydroxybutyrate dehydrogenase) to creatine kinase 2, myoglobin, cardiac troponin T (cTnT), or cardiac troponin I (cTnI), continue because these markers greatly reduce the time required for clinical diagnosis and provide patients effective support. Among them, $\mathrm{cTnT}$ or $\mathrm{cTnI}$, which are derived from heart tissue, are specific markers for myocardial infarction (Zhou and Tu, 2011). cTnT is a cardio-specific marker for myocardial damage (Katus et al., 1989), but its level increases approximately 3-4 hours after AMI (Kawahara et al., 2011). Early detection of the cTnT protein marker in patients with a higher risk of AMI can reduce the risk of death due to heart attacks. However, the clinical use of cTnT is limited by the low sensitivity of conventional commercial assay systems. Therefore, improvements in cardiac markers would enable better diagnosis of AMI. The role of high-sensitivity cTnT (hs-cTnT) in myocardial ischemia has been demonstrated in many studies; hs-cTnT has recently become commercially available (Laufer et al., 2010) and is being produced and distributed by Roche for clinical applications. One report showed that even mild coronary artery disease was associated with quantifiable circulating levels of hs-cTnT in patients without acute coronary syndrome (ACS) (Laufer et al., 2010), whereas other research revealed its function in the prediction of evolving non-ST-segment elevation myocardial infarction among patients with suspected ACS and negative troponin on admission (Giannitsis et al., 2010). However, there is insufficient evidence to support the diagnostic sensitivity and specificity of $\mathrm{cTnT}$ for the detection of myocardial ischemia. The main purpose of this study was to investigate the prognostic value of hs-cTnT in patients with ACS for detecting the recurrence of AMI within 6 months using continuous hs-cTnT detection.

\section{MATERIAL AND METHODS}

Blood samples ( $3 \mathrm{~mL}$ each; without anticoagulants) were collected upon admission, at $6 \mathrm{~h}$ after admission, and during the recovery period from patients with chest pain suspected to be caused by ACS at the Second People's Hospital, Yibin City, Sichuan Province, China, from September 2009 to October 2011. These patients were followed up until February 2012. All patients with suspected AMI were divided into two groups according to ST-segment elevation status on electrocardiography (ECG): the ST-segment elevation myocardial infarction (STEMI) group and the non-STEMI group. The variables $\alpha$ and $\beta$ represent the percentage changes in hs-cTnT concentration from admission time to $6 \mathrm{~h}$ after admission and through the recovery period, respectively. An electrochemical luminescence instrument (e411 ${ }^{\circledR}$; Roche, Basel, Switzerland) was used to test the specimens. The hs-cTnT reagent (REF: 05092728190) is a fifth-generation AMI marker produced by Roche. The data of the control group were collected from 138 healthy persons.

Data are reported as means \pm standard deviation (SD) or as proportions. In cases in which significant differences were observed between groups, multiple comparisons were performed using one-way analysis of variance (ANOVA). An independent $t$-test was used for com- 
parisons between the two groups. All statistical analyses were performed using the SPSS 18.0 software (Chicago, IL, USA).

\section{RESULTS}

The background characteristics stratified by disease for patients are shown in Table 1. The occurrence of ACS was significantly correlated with baseline characteristics such as living habits and lipid serum levels among patients with AMI, unstable angina (UA), heart failure (HF), and the healthy control group.

The determined clinical sensitivity and specificity of hs-cTnT in the 134 patients with AMI are shown in Table 2. As the hs-cTnT concentration increased, the diagnostic specificity increased whereas sensitivity decreased. However, the sensitivity of the $\alpha$ values did not change, and the specificity increased when $\alpha$ increased. Therefore, the change in hs-cTnT concentration between the two detection periods can be used for rapid diagnosis and effective treatment of AMI.

\begin{tabular}{|c|c|c|c|c|c|c|}
\hline \multirow[t]{2}{*}{ Items } & \multicolumn{2}{|c|}{ AMI } & \multirow{2}{*}{$\begin{array}{c}\text { UA } \\
(\mathrm{N}=94)\end{array}$} & \multirow{2}{*}{$\begin{array}{c}\mathrm{HF} \\
(\mathrm{N}=155)\end{array}$} & \multirow{2}{*}{$\begin{array}{c}\text { Control } \\
(\mathrm{N}=138)\end{array}$} & \multirow[t]{2}{*}{$P$} \\
\hline & STEMI $(\mathrm{N}=69)$ & NSTEMI $(\mathrm{N}=65)$ & & & & \\
\hline Gender $(\mathrm{M} / \mathrm{F})$ & $32 / 37$ & $24 / 41$ & $22 / 29$ & $83 / 72$ & $76 / 62$ & NS \\
\hline Age (years) & $67 \pm 13$ & $59 \pm 20$ & $71 \pm 11$ & $68 \pm 12$ & $57 \pm 11$ & NS \\
\hline Smoking (N, \%) & $17(24.6 \%)$ & $21(32.3 \%)$ & $19(37.3 \%)$ & $6(3.87 \%)$ & $5(3.62)$ & $<0.01$ \\
\hline Drinking $(\mathrm{N}, \%)$ & $28(40.6 \%)$ & $35(53.8 \%)$ & $24(47.1 \%)$ & $61(39.4 \%)$ & $9(6.5 \%)$ & $<0.05$ \\
\hline Hypertension (N, \%) & $23(33.3 \%)$ & $17(26.2 \%)$ & $16(31.4 \%)$ & $56(36.1 \%)$ & $3(2.2 \%)$ & $<0.01$ \\
\hline Diabetes mellitus (N, \%) & $9(13.0 \%)$ & $7(10.2 \%)$ & $11(21.6 \%)$ & $31(20.0 \%)$ & $5(3.6 \%)$ & $<0.05$ \\
\hline Triglycerides (mM) & $1.84( \pm 0.36)$ & $1.69( \pm 0.43)$ & $1.77( \pm 0.24)$ & $1.67( \pm 0.44)$ & $1.29( \pm 0.33)$ & $<0.01$ \\
\hline Cholesterol (mM) & $6.69( \pm 1.03)$ & $5.93( \pm 0.83)$ & $6.51( \pm 0.71)$ & $5.41( \pm 1.05)$ & $4.69( \pm 0.74)$ & $<0.01$ \\
\hline High density lipoprotein (mM) & $0.75( \pm 0.31)$ & $0.81( \pm 0.22)$ & $0.84( \pm 0.29)$ & $1.02( \pm 0.26)$ & $1.35( \pm 0.27)$ & $<0.001$ \\
\hline Low density lipoprotein (mM) & $4.15( \pm 1.16)$ & $4.17( \pm 0.83)$ & $3.86( \pm 0.86)$ & $4.09( \pm 1.04)$ & $2.31( \pm 0.53)$ & $<0.001$ \\
\hline
\end{tabular}

Table 2. Clinical sensitivity and specificity of hs-cTnT in 134 patients with acute myocardial infarction.

\begin{tabular}{lcrr}
\hline & hs-cTnT concentration & Sensitivity \% (95\% CI) & Specificity \% (95\%CI) \\
\hline On admission & $>24.9 \mathrm{ng} / \mathrm{L}$ & $72.2(53.1-84.1)$ & $79.1(71.9-86.3)$ \\
At 6 h after admission & $>24.9 \mathrm{ng} / \mathrm{L}$ & $93.8(83.7-98.2)$ & $4.3(78.1-89.4)$ \\
$\alpha$ increased & $>10 \%$ & $76.3(64.1-87.0)$ & $82.9(74.6-86.3)$ \\
& $>20 \%$ & $75.8(63.4-85.2)$ & $85.4(81.1-92.6)$ \\
& $>30 \%$ & $75.9(63.5-89.5)$ & $91.4(85.3-94.7)$ \\
\hline
\end{tabular}

hs-cTnT $=$ high-sensitivity cardiac troponin $\mathrm{T} ; \mathrm{CI}=$ confidence interval

The proportion of patients with AMI and hs-cTnT concentration changes between admission and $6 \mathrm{~h}$ after admission is shown in Table 3. The proportion of patients with AMI diagnosis correlated with the initial hs-cTnT concentration and the change in $\alpha$ values.

We analyzed the concentration of hs-cTnT at admission and during the recovery period after different treatments, such as percutaneous coronary intervention, coronary artery bypass grafting, and thrombolytic therapy, to determine which treatment was more effective for patients with ACS (Table 4).

The occurrence of AMI in the 134 patients during hospitalization and the subsequent one-year follow-up period until February 2012 is shown in Table 5. Within one year, compared with hospitalization period, $\beta$ correlated with ACS reccurrence and the risk of death. 
Table 3. Proportion of patients diagnosed with acute myocardial infarction (AMI) on the basis of changes in hs-cTnT concentration between admission and $6 \mathrm{~h}$ after admission.

\begin{tabular}{lcc}
\hline hs-cTnT concentration & Increase in $\alpha$ & Proportion of patients diagnosed with AMI \\
\hline$<24.9 \mathrm{ng} / \mathrm{L}$ on admission & $\alpha<20 \%$ & $4 / 217(1.84 \%)$ \\
& $20 \%<\alpha<30 \%$ & $6 / 109(5.50 \%)$ \\
& $\alpha>30 \%$ & $27 / 59(45.76 \%)$ \\
$>24.9 \mathrm{ng} / \mathrm{L}$ on admission & $\alpha<20 \%$ & $14 / 86(16.28 \%)$ \\
& $20 \%<\alpha<30 \%$ & $24 / 76(31.58 \%)$ \\
& $\alpha>30 \%$ & $37 / 42(88.09 \%)$ \\
\hline
\end{tabular}

hs-cTnT $=$ high-sensitivity cardiac troponin T. The percentage in parentheses is the occurrence probability of AMI.

Table 4. Baseline parameters of patients with acute myocardial infarction (AMI) undergoing different treatments and the concentration of hs-cTnT during the recovery period.

\begin{tabular}{llcccc}
\hline Admission & & \multicolumn{3}{c}{ Recovery } \\
\cline { 3 - 5 } & & & PCI & CABG & Thrombolytic therapy \\
\hline Patients & STEMI & 69 & 21 & 8 & 40 \\
hs-cTnT & NSTEMI & 65 & 11 & 5 & 49 \\
(ng/L) & STEMI & $576 \pm 143$ & $135 \pm 37.5^{* *}$ & $87.2 \pm 31.1^{* *}$ & $315^{*} \pm 697^{*}$ \\
& NSTEMI & $399 \pm 170$ & $87.4 \pm 43^{* *}$ & $65 \pm 30.9^{* *}$ & $187.2 \pm 81.2^{*}$ \\
\hline
\end{tabular}

hs-cTnT $=$ high-sensitivity cardiac troponin $\mathrm{T} ; \mathrm{PCI}=$ percutaneous coronary intervention; $\mathrm{CABG}=$ coronary artery bypass grafting; STEMI = ST-elevation myocardial infarction; NSTEMI = non-ST-elevation myocardial infarction. Data are reported as means \pm SD. Significant comparisons were performed between patients undergoing different therapy and admission. ${ }^{*} \mathrm{P}<0.01 ; * \mathrm{P}<0.05 ; \mathrm{ns}=$ not significant.

Table 5. Occurrence of acute coronary syndrome (ACS) in patients with acute myocardial infarction (AMI) after different treatment during hospitalization and one-year follow-up.

\begin{tabular}{|c|c|c|c|c|c|}
\hline & $\begin{array}{l}\text { Total } \\
\text { case }\end{array}$ & $\begin{array}{l}\text { Occurred } \\
\text { STEMI }\end{array}$ & $\begin{array}{l}\text { Occurred } \\
\text { NSREMI }\end{array}$ & $\begin{array}{l}\text { Occurred } \\
\text { UA }\end{array}$ & $\begin{array}{c}\text { Accumulated case proportion } \\
\text { in one year, } \%\end{array}$ \\
\hline \multicolumn{6}{|l|}{ Effective treatment } \\
\hline B decreased $>30 \%$ (compared with admission) & 89 & 4 & 4 & 7 & 16.85 \\
\hline \multicolumn{6}{|l|}{ PCI } \\
\hline STEMI & 17 & 0 & 1 & 0 & 5.88 \\
\hline NSTEMI & 8 & 1 & 0 & 0 & 12.50 \\
\hline \multicolumn{6}{|l|}{ CABG } \\
\hline STEMI & 6 & 0 & 0 & 0 & 0 \\
\hline NSTIMI & 3 & 0 & 0 & 0 & 0 \\
\hline \multicolumn{6}{|l|}{ Thrombolytic therapy } \\
\hline STIMI & 26 & 2 & 2 & 1 & 19.28 \\
\hline NSTEMI & 29 & 1 & 1 & 6 & 27.59 \\
\hline \multicolumn{6}{|l|}{ Treatment not satisfied } \\
\hline B decreased $>30 \%$ (compared with admission) & 45 & 6 & 10 & 12 & 62.22 \\
\hline \multicolumn{6}{|l|}{ PCI } \\
\hline STEMI & 4 & 1 & 0 & 1 & 19.23 \\
\hline NSTEMI & 3 & 0 & 1 & 1 & 66.67 \\
\hline \multicolumn{6}{|l|}{ CABG } \\
\hline STEMI & 2 & 0 & 1 & 0 & 50.0 \\
\hline NSTIMI & 2 & 0 & 2 & 1 & 50.0 \\
\hline \multicolumn{6}{|l|}{ Thrombolytic therapy } \\
\hline STIMI & 20 & 2 & 5 & 3 & 50.0 \\
\hline NSTEMI & 25 & 3 & 3 & 6 & 48.0 \\
\hline
\end{tabular}

PCI $=$ percutaneous coronary intervention $; \mathrm{CABG}=$ coronary artery bypass grafting; STEMI = ST-elevation myocardial infarction; NSTEMI = non-ST-elevation myocardial infarction. Significant comparisons were performed in each therapy group within patient groups (decreased $<30 \%$ vs decreased $>30 \%$ ). $* \mathrm{P}<0.05 ; * * \mathrm{P}<0.01$. 


\section{DISCUSSION}

ACS is a group syndrome caused by acute myocardial ischemia, induced by more factors from living habits to physical causes for human beings. It includes AMI and UA. ACS is a common clinical cardiovascular disease with a high incidence, and its early diagnosis and prompt treatment have vital significance in patient outcomes. Therefore, it is necessary to optimize the clinical sensitivity and specificity of the diagnostic methods of AMI.

The new "universal definition of myocardial infarction" advised the redefinition of AMI, which was previously defined by the ESC/ACCF/AHA/WHF (Thygesen et al., 2007). To optimize the diagnostic sensitivity for myocardial infarction while excluding clinical specificity (Morrow et al., 2007), the immediate measurement of cardiac troponin concentrations is recommended upon hospital admission as well as 6-9 $\mathrm{h}$ after admission. These results were consistent with the U.S. National Academy of Clinical Biochemistry (NACB) Laboratory Medicine Practice Guidelines (Apple et al., 2007). Furthermore, based on the NACB recommendations, if linked with the treatment strategy, the markers for myocardial necrosis can be sampled rapidly and frequently. With the improvement of troponin-detection techniques, the detection limits for $\mathrm{cTnT}$ have now reached the nanogram per milliliter level and the precision has reached the 99th percentile. Both diagnostic sensitivity and risk stratification can be improved by monitoring troponin concentrations and including slight changes.

Elevated troponin $\mathrm{T}$ levels correlate with the severity of coronary artery disease and poor outcomes, independent of natriuretic peptide concentrations (Latini et al., 2007; Omland et al., 2008). The results showed that any measurable hs-cTnT concentration is an independent predictor for an adverse event in patients with ACS symptoms (Thygesen et al., 2007). Overall, these findings supported the use of troponin as the biomarker of choice in risk stratification, and NACB advocated and recommended its measurement in all patients with suspected ACS. A troponin concentration above the 99th percentile is an indicator of death and an increased risk of recurrent ischemic events (Giannitsis et al., 2010). Cardiac troponin is the preferred marker for myocardial injury in the new guidelines for the diagnosis and treatment of non-STsegment elevation ACS (Apple et al., 2007). Low troponin T concentrations can be detected in clinically stable patients, such as those with ischemic or non-ischemic heart conditions (Shave et al., 2007; Giannitsis et al., 2010).

Our results show that hs-cTnT is a predictor of myocardial disease, especially for patients with poor lifestyle habits. The hs-cTnT concentration is much higher in ACS patients than that detected in patients with other diseases, and its sensitivity facilitates the detection of transient cardiac injury. These results provide evidence that hs-cTnT is an appropriate biomarker for detecting UA, which is usually detected through transient ECG changes. In addition, the emergence of hs-cTnT detection kits in clinical trials may facilitate the detection of hs-cTnT in patients with particular diseases, especially with an analytical range of 3-10,000 $\mathrm{ng} / \mathrm{L}$ and coefficient of variation at $9 \%$ using the Elecsys e 411 analyzer. The assay was specific for cTnT without interference from human cTnI, cTnC, skeletal muscle TnT, and hemoglobin concentrations up to $1,000 \mathrm{mg} / \mathrm{L}$, above which false lower values could be expected (Mingels et al., 2009). The increase in hs-cTnT concentrations in normal controls is $<1 \%$, whereas these concentrations are increased significantly in patients with HF (Alehagen et al., 2010), diabetes mellitus, and ventricular hypertrophy due to unbalanced blood supply and demand. Hence, hs-cTnT is a critical factor for quantifying and following up chronic myocardial ischemia. 
This could account for the obvious increase in hs-cTnT concentrations in HF (Shave et al., 2007). In addition, it is a useful prognostic predictor independent of left ventricular ejection fraction or B-type natriuretic peptide in patients with congestive HF and non-ischemic dilated cardiomyopathy. In sepsis, measurements of circulating hs-cTnT may be an early marker for shock (Røsjø et al., 2011). These results demonstrate that hs-cTnT is a much more sensitive and timely detector for AMI than cTnT. Moreover, it effectively monitors transient cardiac injury, especially in marathon runners, thus leading to the clinical detection of cardiovascular diseases (Saravia et al., 2010).

Although the function of hs-cTnT in AMI prognosis requires further investigation, the current study provides evidence that hs-cTnT improves the sensitivity of AMI diagnosis and is more effective in risk stratification of adverse events in patients with suspected ACS. Moreover, increase in the $\alpha$ value is an important indicator of AMI diagnosis.

When hs-cTnT was $>30 \mathrm{ng} / \mathrm{L}$, the median diagnostic sensitivity for MI was $69 \%$ within $4 \mathrm{~h}$ after the onset of MI. The diagnostic sensitivity of the second detection reached $94 \%$, in which the risk of cardiac events or death was four times greater than that at the initial hs-cTnT concentration of $>30 \mathrm{ng} / \mathrm{L}$ in patients after 60 days (Lindahl et al., 2010). Specificity is lower when detection sensitivity of hs-cTnT increases. Accordingly, the specificity in our study was only $48.3 \%$ when the sensitivity was $92.4 \%$. After treatment, a response and $>75 \%$ increase in hs-cTnT indicates that the test is an independent prognostic determinant, a finding that is consistent with the findings of Palladini et al. (2010). The diagnostic performance of sensitive cardiac troponin assays is convincing, and these assays can substantially improve the early diagnosis of AMI, particularly in patients with recent-onset chest pain (Reichlin et al., 2009). In conclusion, there is a high risk of ACS reoccurrence and death in patients whose hs-cTnT concentration during the recovery period increases by $>30 \%$ or decreases by $<30 \%$ compared with the concentration at admission during one-year follow-up.

Our results suggest a significant correlation between the occurrence of AMI and patients' lifestyle habits. Changes in hs-cTnT concentration at admission and at $6 \mathrm{~h}$ after admission in patients with ACS have good sensitivity and specificity for the diagnosis of AMI and constitute a better indicator for AMI. In patients with pre-existing cardiovascular disease after discharge, changes in hs-cTnT during the recovery period have better prognostic value than changes in hs-cTnT upon admission. Therefore, hs-cTnT is a sensitive and specific biomarker for AMI in patients with ACS, and the risk assessment can be improved when changes in hs$\mathrm{cTnT}$ concentration are monitored.

\section{ACKNOWLEDGMENT}

Research supported by the Major Science and Technology Project of YiBin (\#200903003).

\section{REFERENCES}

Alehagen U, Dahlstrom U, Rehfeld JF and Goetze JP (2010). Prognostic assessment of elderly patients with symptoms of heart failure by combining high-sensitivity troponin $\mathrm{T}$ and $\mathrm{N}$-terminal pro-B-type natriuretic peptide measurements. Clin. Chem. 56: 1718-1724.

Apple FS, Jesse RL, Newby LK, Wu AH, et al. (2007). National Academy of Clinical Biochemistry and IFCC Committee for Standardization of Markers of Cardiac Damage Laboratory Medicine Practice Guidelines: Analytical issues for 
biochemical markers of acute coronary syndromes. Circulation 115: e352-e355.

Giannitsis E, Becker M, Kurz K, Hess G, et al. (2010). High-sensitivity cardiac troponin T for early prediction of evolving non-ST-segment elevation myocardial infarction in patients with suspected acute coronary syndrome and negative troponin results on admission. Clin. Chem. 56: 642-650.

Katus HA, Remppis A, Looser S, Hallermeier K, et al. (1989). Enzyme linked immuno assay of cardiac troponin T for the detection of acute myocardial infarction in patients. J. Mol. Cell Cardiol. 21: 1349-1353.

Kawahara C, Tsutamoto T, Nishiyama K, Yamaji M, et al. (2011). Prognostic role of high-sensitivity cardiac troponin T in patients with nonischemic dilated cardiomyopathy. Circ. J. 75: 656-661.

Latini R, Masson S, Anand IS, Missov E, et al. (2007). Prognostic value of very low plasma concentrations of troponin T in patients with stable chronic heart failure. Circulation 116: 1242-1249.

Laufer EM, Mingels AM, Winkens MH, Joosen IA, et al. (2010). The extent of coronary atherosclerosis is associated with increasing circulating levels of high sensitive cardiac troponin T. Arterioscler. Thromb. Vasc. Biol. 30: 1269-1275.

Lindahl B, Venge P and James S (2010). The new high-sensitivity cardiac troponin T assay improves risk assessment in acute coronary syndromes. Am. Heart J. 160: 224-229.

Mingels A, Jacobs L, Michielsen E, Swaanenburg J, et al. (2009). Reference population and marathon runner sera assessed by highly sensitive cardiac troponin T and commercial cardiac troponin T and I assays. Clin. Chem. 55: 101-108.

Morrow DA, Cannon CP, Jesse RL, Newby LK, et al. (2007). National Academy of Clinical Biochemistry Laboratory Medicine Practice Guidelines: clinical characteristics and utilization of biochemical markers in acute coronary syndromes. Clin. Chem. 53: 552-574.

Omland T, DE Lemos JA and Christophi C (2008). On behalf: PEACE investigations. Distribution and determinants of very low cardiac troponin $\mathrm{T}$ in patients with stable coronary artery disease: The PEACE trial. Eur. Heart J. 9: 1342.

Palladini G, Barassi A, Klersy C, Pacciolla R, et al. (2010). The combination of high-sensitivity cardiac troponin T (hs$\mathrm{cTnT}$ ) at presentation and changes in N-terminal natriuretic peptide type B (NT-proBNP) after chemotherapy best predicts survival in AL amyloidosis. Blood 116: 3426-3430.

Reichlin T, Hochholzer W, Bassetti S, Steuer S, et al. (2009). Early diagnosis of myocardial infarction with sensitive cardiac troponin assays. N. Engl. J. Med. 361: 858-867.

Røsjø H, Varpula M, Hagve TA, Karlsson S, et al. (2011). Circulating high sensitivity troponin T in severe sepsis and septic shock: distribution, associated factors, and relation to outcome. Intensive Care Med. 37: 77-85.

Saravia SG, Knebel F, Schroeckh S, Ziebig R, et al. (2010). Cardiac troponin T release and inflammation demonstrated in marathon runners. Clin. Lab. 56: 51-58.

Shave R, George K and Gaze D (2007). The influence of exercise upon cardiac biomarkers: a practical guide for clinicians and scientists. Curr. Med. Chem. 14: 1427-1436.

Thygesen K, Alpert JS and White HD (2007). Universal definition of myocardial infarction. J. Am. Coll. Cardiol. 50: 2173-2195.

Zhou X and Tu ZG (2011). The Clinical Biochemistry and Biochemical Tests (3rd edn). People's Medical Publishing House, Beijing. 